\title{
Bio-psycho-social-spiritual needs of adolescents and young adults with life-threatening illnesses: Implications for social work practice
}

Emily Beerbower, BSW, MSW, LSW, Drew Winters, MSW, and David Kondrat, PhD

\section{ABSTRACT}

This paper explores the biopsychosocial and spiritual needs of adolescents and young adults (AYA) with life-threatening or terminal illnesses. AYA are situated between childhood and adulthood (ages 15-25) and have distinct biopsychosocial and spiritual needs unique to their developmental stage. Having a life-threatening or terminal illness directly challenges normal AYA developmental tasks and identity formation. AYA experience more troubling physical symptoms during the dying process compared to other age groups, which leads to significant psychological distress and an increased need for pharmacological treatments. In general, AYA desire to be fully informed and involved in the health care decision-making process, leading to ethical dilemmas when the AYA is a minor and their wishes differ from the wishes of their legal guardian(s). Social workers are especially well-equipped to serve this population due to aligning professional standards and ability to advocate for holistic care within interdisciplinary teams. Additional research is needed to tailor holistic interven-tions to meet the needs of this population.

\section{KEYWORDS}

Adolescent; end-of-life care; hospice social work; life-threatening illness; young adult

Life-threatening illnesses or terminal illness impacts persons of every age, including adolescents and young adults (AYA). While researchers have explored the impact of terminal illness on people of all ages, less emphasis has been placed on AYA (Bates \& Kearney, 2015). To date, no review of research on AYA, with a wide range of terminal illnesses, has been published. Yet this group has a myriad of biopsychosocial and spiritual needs. Understanding these needs will help in the holistic treatment of AYA with life-threatening illnesses.

Medical advances continue to extend the lives of children with conditions that have been historically fatal during childhood and into adolescence and young adulthood (Fahlberg, 2016). As such, it is extremely important to distinguish AYA to determine how their diagnoses fits into their emerging identities, to what extent their illness defines them as a unique population, 
and to explore how to address their needs in a manner that is appropriate to their developmental level. To address these gaps in our understanding, this paper critically reviews the scholarly literature on the psychosocial, physical, and spiritual needs of AYA youth ages 15-25 and considers implications for psychosocial treatment and end-of-life care.

Social workers are in an especially well-suited position to assist and empower this population on several different levels. For example, social workers may engage AYAs with life-threatening illnesses through direct care and services, emphasizing holistic care and facilitating transparency in conversations in inter-disciplinary teams, and advocating for health care policies that focus on the strengths of AYA with life-threatening illnesses, as well as the gaps in knowledge and interventions.

\section{Population}

AYA fit into a unique phase on the development scale because they are neither children nor adults. During this developmental stage, AYA move toward independence, identity formation, autonomy, and adult responsibilities (Osgood, Foster, \& Courtney, 2010). However, in the United States, AYA are typically "semi-independent" (Osgood et al., 2010), meaning AYA are not yet financially independent and still rely on their families for support. Individuals in this age group may be continuing educational pursuits or developing their early careers (Osgood et al., 2010). These issues are especially highlighted in AYA with life-threatening illnesses as many of these tasks are interrupted, or fail to even be initiated, due to the diagnosis of a serious illness (Barr, Ferrari, Ries, Whelan, \& Bleyer, 2016).

Despite recent contributions to the literature surrounding issues facing children with life-threatening and terminal illnesses, little research is available that explores the unique challenges AYA experience due to their developmental stages. This gap is especially challenging because different sources define AYA differently. The age range that defines AYA is inconsistent, and AYA are often lumped into studies of younger children and mid-aged adults with life-threatening illnesses (Geiger \& Castellino, 2011; Quinn, Gonçalves, Sehovic, Bowman, \& Reed, 2015). Findings from research describing AYA are so varied that they cover three different decades (Linebarger, Ajayi, \& Jones, 2014). Geiger and Castellino (2011) further emphasize the discrepancies in defining AYA in medical literature not only creates a barrier to reviewing literature, but also may lead to poorer outcomes. This dilemma makes it difficult to draw conclusions about the implications of research on AYA specifically when the research includes children and adults from other age groups. For this paper, AYA is defined as a person from age 15 to 25 years old, as this is the most commonly used age range in the literature on this topic. 
More than 3,000 AYA die from serious illness each year in the United States (Freyer, 2004). These illnesses typically fall into four categories (ACT, 2003). The first category is illnesses that are curable, but will result in death if treatment fails (ACT, 2003). Cancer is an example of an illness that falls under this category (ACT, 2003; Feudtner et al., 2001). The second category is illnesses that can be managed through intensive treatments with the goal of prolonging life, but will eventually lead to death despite aggressive medical treatment (ACT, 2003). Cystic fibrosis (Kazmerski et al., 2016) and Duchenne muscular dystrophy (Janisch, Nolte-Buchholtz, \& von der Hagen, 2016) are illnesses that follow this pattern (ACT, 2003). The third category is progressive conditions that are treated by addressing symptoms and quality of life. These conditions are always fatal, but the dying process can take several years (ACT, 2003). Batten's disease is an example of an illness in this category (ACT, 2003). Finally, conditions resulting in severe neurological disabilities that weaken the person's ability to fight common health conditions leading to death are another illness category AYA may face (Feudtner et al., 2001). One example of this is pneumonia leading to death in an AYA with cerebral palsy.

\section{Developmental considerations}

The diagnosis of a life-threatening or terminal illness in AYA significantly disrupts normal developmental tasks, such as autonomy or identity forma-tion (Barr et al., 2016; Bates \& Kearney, 2015; Davies, Kelly, \& Hannigan, 2015; Freyer, 2004; Greydanus, 2016). AYA who are dying experience conflict between the dying process and their physical, psychological, and social changes (Klepping, 2012). The dying process also has a negative effect on developmental tasks typical to AYA, such as sexuality, relationships, and body image (Barr et al., 2016; Bates \& Kearney, 2015; Knapp, Quinn, Murphy, Brown, \& Madden, 2010).

Adolescence and young adulthood is a developmental period in which AYA move toward independence and autonomy (Davies et al., 2015). In typically developing AYA, this is a time period of emerging independence, identity formation and exploration of future goals and plans (Clark \& Fasciano, 2015). AYA diagnosed with a life-threatening or terminal illness experience feelings of "isolation, vulnerability, dependence, and uncertainty" (Clark \& Fasciano, $\underline{2015}$, p. 102). As a result of being faced with a life-threatening illness, AYA may experience conflict with the developmental stages and tasks of healthy development because they are forced to abandon planning for adulthood, such as education or career plans, and instead focus on their fragile health state (Davies et al., 2015; Stern et al., 2010).

Despite the similarities between palliative care priorities in adults and AYA, the differences in bio-psycho-social-spiritual and developmental 
needs require specialized training that acknowledges these differences and their implications (Wiener, Weaver, Bell, \& Sansom-Daly, 2015). Despite some AYA's developmental and physical resemblance to adults, research has consistently shown the human brain continues to develop into the mid 20's (Clark \& Fasciano, 2015; Winters \& Beerbower, 2017). AYA experience dramatic physical and social changes as they move from childhood into adulthood (Klepping, 2012).

\section{Adjusted developmental tasks and identity formation}

Terminally ill AYA have different challenges from those faced by children and adults who are terminally ill. A life-threatening or terminal diagnosis can interrupt normal developmental tasks creating a barrier for transition from childhood to adolescence and young adulthood (Freyer, 2004). When an AYA is diagnosed with a life-threatening or terminal illness, normal devel-opment must be abandoned (Barr et al., 2016; Davies et al., 2015) and new developmental tasks emerge that revolve around the AYA attempting to form their identity in the context of a shortened life-span (Davies et al., 2015; Stillion \& Papadatou, 2002). AYA diagnosed with a life-threatening or terminal illness try to figure out how their diagnosis fits into their identity and to what extent their illness defines them (Linebarger et al., 2014). AYA often begin to frame their identity in terms of a shortened lifespan, versus having a full life to develop, shape, and change their personal identity (Stillion \& Papadatou, 2002). Common developmental tasks, such as con-tinuing education, dating, and employment are frequently halted by the disease process (Barr et al., 2016). The monumental impact having a life-threatening illness has on normal development frequently leads to develop-mental regression (Barr et al., 2016).

Identity formation is complex for AYA without a life-threatening illness. Adding the terminal diagnosis negatively impacts the AYA's interpersonal relationships, physical development, ability to socialize, and self-view (Knapp et al., 2010). AYA with terminal and life-threatening illnesses may be extre-mely conscious of losing out on the adulthood their peers are approaching and never being able to achieve adult status, independence, and autonomy themselves (Davies et al., 2015; Greydanus, 2016). AYA who receive a terminal diagnosis have to grieve the loss of the future they have envisioned for themselves, as well as the future that friends and families expect for them (Knox et al., 2017). Common rite of passage milestones for AYA, such as going away to college, often are unobtainable for AYA with a life-threatening or terminal illness, yet the youth watch their healthy peers reaching these milestones knowing they cannot (Knox et al., 2017).

AYA's fight for independence can be particularly challenging to balance when they are in the final stages of a disease, because many must return to 
relying on their parents to meet their physical needs (Greydanus, 2016; Grinyer \& Thomas, 2004). As their disease progresses, the AYA may have to rely on another individual for basic self-care, such as bathing and toileting, which can lead to humiliation. This desire to be independent and autonomous until death may lead an AYA to prefer nursing care at home for their end-of-life needs versus having parents, extended family, and/or friends help, especially when it comes to personal care (Grinyer \& Thomas, 2004; Pritchard, Cuvelier, Harlos, \& Barr, 2011).

Social workers can assist an AYA who is dying by acknowledging and honoring their growing need for independence, need for autonomy, developmental level, sexuality concerns and being comfortable having frank but compassionate discussions about death and end-of-life care (Kirk \& Fraser, 2014). Bates and Kearney (2015) assert that failure to create an environment where the AYA feels they can talk about their illness and prognosis openly may lead to an increase in mental health concerns in the youth (Bates \& Kearney, 2015).

\section{Biological needs}

AYA typically report more physical pain than children or adults and require more pharmacological intervention than other populations (Hughes et al., 2015). These physical symptoms tend to be harder to control in AYA than they are with children and adults (Hughes et al., 2015; Mack et al., 2016; Quinn et al., 2015). When AYA who have life-threatening illnesses report refractory pain, the pain is often due to combined physical, spiritual, and psychosocial factors, rather than just the youth's underlying disease process, and may improve with holistic approaches that address all facets of pain (Klepping, 2012).

Most AYA who enter hospice programs experience extensive physical symptoms and require complex symptom management that may include IV nutri-tion, palliative chemotherapy, terminal sedation, oral pain medication, anti-epileptic drugs, and psychosocial services which are required at a more inten-sive level than other age groups (Belasco, Danz, Drill, Schmid, \& Burkey, 2000). The most common physical symptoms AYA experience at the end-of-life from advanced cancer are pain and dyspnea (Cohen-Gogo et al., 2011). Untreated physical symptoms can cause extreme distress and mental health crises among AYA, including suicidal ideation (Bates \& Kearney, 2015).

Pain and significant discomfort must be controlled before AYA can benefit from psychosocial interventions (Jones, 2005; Jones, Christ, \& Blacker, 2006). Despite their seemingly medical causes and treatments, pain and symptom control can, and should, be addressed by social workers (Jones et al., 2006). Interventions that treat AYA holistically and acknowledge the mind-body connection tend to be the most successful in addressing both psychological 
and physical pain (Klepping, 2012). Social workers can help AYA approach pain in healthy ways including alternative pain relief, such as meditation (Jones et al., 2006).

\section{Psychological needs}

AYA facing a life-threatening or terminal diagnosis frequently experience significant anxiety and depression (Bates \& Kearney, 2015; Hølge-Hazelton, Timm, Graugaard, Boisen, \& Sperling, 2016). Despite the high incidence of depression and anxiety in terminally ill AYA, no screening tool exists to assess anxiety and depression in this population (Clark \& Fasciano, 2015). Symptoms of depression often go unrecognized due to the lack of appropriate screening measures and interventions (Hughes et al., 2015). Anxiety typically increases as death approaches and a significant percentage of AYA are prescribed anxiolytic medications at the time of their death (Bates \& Kearney, 2015; Bell, Skiles, Pradhan, \& Champion, 2010). The use of anxiolytic medications for anxiety increase as the age and development of the AYA increases (Bell et al., 2010).

Psychological symptoms can be decreased by allowing AYA as much independence and autonomy at the end-of-life as possible (Cohen-Gogo et al., 2011). Coping skills critical to helping AYA manage psychological and physical symptoms, including severe anxiety, are most successfully implemented with principles of cognitive behavioral therapy, behavioral activation, and coping strategies (Clark \& Fasciano, 2015).

Social workers are able to provide intensive therapy for AYA with lifethreatening illnesses and have expertise in addressing the psychosocial components of the disease (NASW, 2004). Therapy is an important component of hospice and palliative care for AYA and must address the developmental stage of the patient (Brown \& Sourkes, 2006). In addition to addressing anxiety and depression, psychotherapy can help terminally ill AYA address common concerns around grief, loss, sexuality, physical symptoms, and anticipatory grief (Bell et al., 2010; Brown \& Sourkes, 2006).

Psychotherapy can be useful in tackling underlying issues, such as anger and lack of control, that lead AYA to be labeled as difficult or non-compliant by their doctors and medical teams. This may be demonstrated by refusing or lying about taking medications or not adhering to restrictive medically necessary precautions. Often youth are simply reacting in a developmentally appropriate way to the challenges of their illness and may protest as a way to assert their independence (Brown \& Sourkes, 2006; Jones \& Weisenfluh, 2003). Social workers should be mindful that stigma associated with experiencing psycholo-gical challenges, such as being perceived as weak, may prevent AYA from engaging in services (Creswell, Christie, \& Boylan, 2001). Thus, creative and empowering strategies to engage AYA while minimizing stigma are important. 
Social workers can be instrumental in helping AYA complete emotional end-of-life tasks, such as writing letters to family and friends (Zadeh, Pao, \& Wiener, 2015). AYA face several unique challenges that may contribute to or complicate mental health concerns. Coping skills, in regards to painful physical and psychological symptoms, are vitally important. Psychotherapy that introduces AYAs to creative and nonpharmacological coping skills, such as guided imagery, massage, and expressive arts tend to be effective (Jones et al., 2006; Knapp et al., 2010; Knox et al., 2017; Pritchard et al., 2011).

\section{Role of communication in psychological well-being}

Poor communication is often a source of anxiety for AYA, and intensifies when information about prognosis and treatment is withheld by family and medical providers (Bates \& Kearney, 2015). AYA consistently report not being provided information about their disease, treatment, and prognosis, which exacerbates psychological symptoms (Bates \& Kearney, 2015). Likewise, a lack of trust can create a barrier to the success of psychological interventions (Wein, Pery, \& Zer, 2010). In contrast, AYA who are given accurate information and have open communication with their medical providers report less anxiety (Lyon, Jacobs, Briggs, Cheng, \& Wang, 2014). Social workers should recognize the need for AYA to have information about their medical condition and prognosis, as well as the need for autonomy and control in their end-of-life decision-making (Jones et al., 2006). In addition, social work's expertise in communication can assist the AYA in expressing needs and concerns with their medical providers. Social workers also serve as a liaison between patients and medical providers (Jones, 2005).

\section{Managing distressing physical and psychological symptoms}

As previously discussed, AYA have worse treatment outcomes and more troubling symptoms at the end-of-life when compared to other age groups, which can have a dramatic impact on the youth's quality of life (Klepping, 2012). Unfortunately, aggressive treatment for the primary disease at the end-of -life usually leads to substantial, secondary, iatrogenic symptoms (Stillion \& Papadatou, 2002). It is extremely important for providers to address these iatrogenic symptoms holistically from a quality of life perspective. For example, bone pain from diseases, uncertainty about what happens after death, loss of friendships, and feelings of helplessness all make up the AYA's physical and psychological pain and affect quality of life (Klepping, 2012). Troublesome symptoms that impact quality of life must be treated early and aggressively since AYA frequently experience significant symptoms despite aggressive med-ical intervention meant to relieve suffering (Hughes et al., 2015). 


\section{Social needs}

Social interaction and peer relationships are extremely important to AYA (Bates \& Kearney, 2015; Knox et al., 2017). AYA with life-threatening illnesses frequently experience severe social isolation because their disease and sometimes embarrassing symptoms, prevents them from making new friends and sustaining previous friendships (Carter, Qualter, \& Dix, 2015). Despite this, pursuing typical adolescent experiences remain a priority regardless of the underlying illness or the AYA's life expectancy (Bates \& Kearney, 2015).

AYA frequently make friends with other youth undergoing treatment. When their friends with similar illnesses die, the loss is especially significant (Knapp et al., 2010; Marris, Morgan, \& Stark, 2011) since it reminds AYA that they too may have a shortened life-span and may lead to questioning why they survived when their friends did not. This loss may also occur when the AYA leaves curative treatment and moves on to hospice care while their friends remain on a curative path (Pritchard et al., 2011).

The impact of serious illness and treatment on sexuality is profound (Barr et al., 2016). However, sexual development and health is crucial in AYAs but is often ignored for AYAs with serious illness (Bakke, 2016) or is discussed only in the context of fertility (Morgan, Davies, Palmer, \& Plaster, 2010). Despite a life-threatening diagnosis, AYA typically have a similar degree of interest in discovering their sexuality as their healthy peers (Bates \& Kearney, 2015). As such, AYAs with serious illnesses should be viewed by their providers as similar to healthy peers in terms of developing sexuality (Bakke, $\underline{2016)}$.

\section{Spiritual needs}

Hospice programs always incorporate spirituality into their offered services. However, these needs are frequently given significantly less attention than biopsychosocial needs (Kelly, May, \& Maurer, 2016). Hospice care tends to focus solely around religious practice and preference, leaving holistic spirituality unaddressed (Davies, Brenner, Orloff, Sumner, \& Worden, 2002). In pediatric hospice and palliative care, religious and spiritual practices are usually asked of the parent, instead of the child, further distancing spiritual involvement in the child's end-of-life treatment plan (Davies et al., 2002). This is especially problematic in providing services to AYA as they may have developed belief systems that are different from their parent's. Conversations about spirituality with AYA should always open with what spirituality means to them (McNeil, 2016).

Spiritual interventions aim "to ease, resolve, or reconcile spiritual pain and suffering so patients may experience peace, reconciliation, wholeness, and comfort" (Jones \& Weisenfluh, 2003, p. 436). Discussions surrounding 
spirituality must be addressed and implemented at the patient's developmental level. Journaling, poetry, writing stories and answering guided questions about death, dying, and spirituality in writing can be particularly effective for AYA (Davies et al., 2002). Spiritual suffering often presents as anxiety about death, inability to make meaning, and hopelessness (Foster, Bell, \& Gilmer, 2012). Unlike physical and/or psychological suffering, symptoms of spiritual suffering in children and AYA may be less pronounced and are less likely to be addressed (Foster et al., 2012).

\section{Spirituality versus religion in end-of-life care}

Spirituality, and its application in pediatric hospice, expands outside of the patient's religious preference (Davies et al., 2002). Despite the definition's lack of reference to organized religion, most writings focus on the theological understanding and application of spirituality (Davies et al., 2002). In pediatric hospice and palliative care, it is imperative that providers are able to recognize the differences and similarities between religion and spirituality, including understanding religious customs (Davies et al., 2002). Youth and their families are frequently more familiar with religious preferences. Discussing religious practices can be used as a gateway into discussions about spiritual needs (Davies et al., 2002). AYA with terminal illness fre-quently struggle to incorporate and frame religion into their illness and early death (Linebarger et al., 2014). Young adults with terminal illness typically report religion and spirituality as important but rank the actual practice of religion as less important (Clark \& Fasciano, 2015).

When working with AYA receiving hospice care, providers should remember that discussions surrounding spirituality should be ongoing and progressive, enhanced by the level of relationship the worker has with the child (Davies et al., 2002). Spiritualty in this population is not a linear experience and reflects the individual's journey through the course of their illness (Foster et al., 2012).

\section{Quality of life during the dying process}

Quality of life is a critically important issue for AYA who are seriously ill or dying, and little research explores the quality of life specific to this group (Bell et al., 2010). Quality of life provides a holistic lens, including, psychological, social, relational, environmental, and spiritual dimensions of a person's life. Little understanding exists as to how a terminal or life-threatening illness affects AYA's quality of life. When AYA receive a terminal diagnosis, they must begin to plan for how they want to live the remainder of their lives, including how they want to die. Despite the vast majority of AYA and their families wishing for death to occur at home, most AYA die in a hospital 
setting (Bell et al., 2009). In fact, in one study, almost half of AYAs who died in a hospital died in the intensive care unit (Bell et al., 2009). Patients and families express the need to feel safe in all aspects (Jones et al., 2006) as they enter the end-of-life and social workers can meet this need by being present and "bearing witness" to the AYA's journey throughout their illness and treatment (Jones, 2005; Jones et al., 2006).

\section{End-of-life discussions and advanced care planning}

The NASW supports the right of individuals to determine their end-of-life care with special attention to vulnerable populations, including children (NASW, 2004). AYA frequently have no knowledge about hospice or advanced directives prior to needing these services (Jacobs et al., 2015). However, AYA want to know their prognosis and to be involved in decisions about their end-of-life care (Bates \& Kearney, 2015). Despite the desire to be involved in end-of-life decisions making, many barriers exist limiting AYAs involvement. AYA are frequently viewed as strong and at the peak of their life from a physical standpoint. Many pediatric health care settings create a "culture of hope," reflecting AYA's resiliency and ability to survive (Wiener et al., 2008, p. 1309). While hope is important, understating the reality of an AYA's condition can result in difficulties initiating end-of-life discussions as it seemingly counteracts the culture of a pediatric healthcare setting (Wiener, 2012). AYA should have accurate medical information about their condition, prognosis, and treatment options throughout their entire course of treat-ment, enabling the end-of-life conversations to be an ongoing dialogue between the youth, their family, and their medical providers.

One barrier to end-of-life discussions with AYA and their families is the medical teams' discomfort, and lack of training and confidence in discussing dying with patients, especially young patients (Bates \& Kearney, 2015; Kazmerski et al., 2016). Health care providers may also be reluctant to stop treatment, hoping for a cure despite diagnostic evidence of treatment failure (Bates \& Kearney, 2015). Similarly, medical providers struggle dealing with their own emotions surrounding their young patients' impeding death. From the beginning of treatment, providers desire to protect AYA and family members from the terminal prognosis, resulting in end-of-life discussions occurring close to the time of death (Bell et al., 2010). When end-of-life discussions do take place, parents are given the information first and the information is filtered from the parent to the patient (Bates \& Kearney, 2015).

This broken system of communication can cause additional stress and delays for the youth. The broken communication is so significant that more than half of AYAs dying of cancer do not discuss end-of-life issues until their final month of life, which is not an adequate amount of time for the AYA and 
their families to prepare for death (Bell et al., 2010). Likewise, the lack of disclosure about the youth's prognosis contributes to increased anxiety and a breakdown in trust between the youth, their parents, and their medical team (Bates \& Kearney, 2015; Lyon et al., 2014). Early communication is essential to prevent conflict when a medical crisis occurs.

\section{Ethics}

There are multiple, complex ethical dilemmas and issues facing AYA with life-threatening and terminal illnesses. One ethical issue concerns AYA participating in research studies since they are considered a protected and vulnerable population. AYA are often asked to consent to research due to the substantial needs to understand this population and their needs, but researchers frequently run into barriers gaining approval for such studies (Hinds, Burghen, \& Pritchard, 2007). Ethical issues also arise when decisions about end-of-life care are made, especially when the AYA is still legally a minor.

Another ethical issue is whether or not, and to what extent, AYA under the age of 18 should be allowed to make medical decisions about their end-of -life care. The United States does not currently grant minors any legal rights to make medical decisions concerning their care (Stillion \& Papadatou, 2002). In rare cases a court can deem the AYA is a "mature minor" after determining their competency allowing for the AYA to make their own decisions about their health care despite not being a legal adult (Derish \& Heuvel, 2000).

Ethical dilemmas occur when there is disagreement between the youth, their family (including difference of opinions between parents), and the medical team regarding stopping treatment, transparency in prognosis, and palliative mea-sures (Muirhead, 2004). Failure to have an open dialogue between family members, youth, and/or medical team members about the AYA's treatment and prognosis creates an ethical dilemma that has real life implications for all involved. Social workers can work with the AYA, family and medical team to have these hard, but necessary conversations.

\section{Gaps in literature}

One gap in the literature is that most research focuses on AYA with diagnoses of cancer, excluding other life-threatening illness (Fraser, Harris, Berringer, Prescott, \& Finlay, 2010). Since different life-threatening and terminal disease progressions produce different symptoms, research is needed across the spectrum of life-threatening illnesses because it can be assumed different issues arise for AYA who have had a life-threatening illness since birth and AYA who grew up healthy and were diagnosed with 
cancer later in life. When exploring medical, physical, and psychosocial needs of AYA who are dying, understanding how to control symptoms and address physical needs attributed to a diverse group of illnesses is paramount to successful treatment.

Another gap is exploring if there is a difference in outcomes between AYA treated in pediatric hospital versus AYA who continue their care in pediatric hospitals. This is especially relevant for AYA that began receiving services in a pediatric hospital and had to transition to the adult health care system, due to their increasing age, as this may create additional hardship. More research, and understanding, about this gap may lead to changes in policy for AYA "aging out" of pediatric healthcare, either allowing them to stay with their pediatric teams, or affirming transfer to adult health care is in their best interest.

Multiple definitions for AYA, hospice care, and palliative care prove to be problematic. Palliative care and hospice care are often used interchangeably, despite the fact hospice care is reserved for patients with an expected lifeexpectancy of 6 months or less while palliative care is aimed at reducing suffering for patients who have complex needs and symptoms who may not have an illness that is expected to result in death within 6 months. Terms are also used differently across cultures, with hospice having a broader meaning in the UK than it does in the US. The interchangeability and ambiguity of these terms can create confusion when understanding the type of treatment the AYA is going to receive. Using the term hospice when meaning palliative may lead AYA and family members to misunderstand the prognosis of an illness and the purpose of care.

Likewise, there are several different definitions of the age group that encompasses AYA. Other research may include AYA's responses in studies that also include young children without differentiating between their developmental stages and how that impacts research results when multiple developmental stages are lumped into a single study. In order to develop targeted treatments, including psychosocial treatment, the research and treatment communities must come to a consensus about the age range for AYA.

There is also a lack of research regarding care specifically designed for AYA (Kirk \& Fraser, 2014) especially in the form of evidenced-based practices (Pritchard et al., 2011). Specifically, there is little research exploring how the different developmental stages that occur within adolescent and young adulthood effect and influence the dying process (Bell et al., 2010). More research is required to understand this population in all its diversity.

Implications for social work practice with AYA: social work in end-of-life care

The National Association of Social Workers (NASW, 2004) recognizes social work's valuable role in end-of-life care and has released standards for 
palliative and end-of-life care. The standards address ethics and values, knowledge, assessment, intervention/treatment planning, attitude/selfawareness, empowerment and advocacy, documentation, interdisciplinary teamwork, cultural competence, continuing education, and supervisions, leadership, and training (NASW, 2004). Social workers should possess an understanding about the framework of the medical systems and the policies and programs impacting AYA and their families (Jones \& Weisenfluh, 2003). They should be able to assist AYAs and their families in navigating the medical system and surpassing system barriers (NASW, 2004). Social workers should possess knowledge about the AYA's culture and how cultural tradi-tions and beliefs impact the dying process. Social workers can then use this knowledge to advocate for AYA's to be able to incorporate important cultural components in their care and following their death regardless of hospital policies (Jones \& Weisenfluh, 2003).

Social workers are especially qualified to assess and meet bio-psychosocial -spiritual needs of AYA and their families (Bosma et al., 2010; Jones \& Weisenfluh, 2003). Social work's broad professional base and knowledge in cultural competency, interventions across the life cycle, assessment, and multidisciplinary practice make the profession especially valuable in end-oflife care (Bosma et al., 2010; NASW, 2004). Families who have children and AYAs with life-threatening illnesses often face additional social challenges and needs (Beaune et al., 2014). These needs frequently go unnoticed by other members of the health care team or the team is not sure how to address them (Beaune et al., 2014; Remke \& Schermer, 2012). Social workers are able to fill this gap on interdisciplinary teams due to their expertise and knowl-edge of resources that address unmet needs as well as encourage other members of the health team to screen families for additional needs impacting the AYA (Beaune et al., 2014).

Social workers have a critical role on interdisciplinary teams which benefit AYA (Remke \& Schermer, 2012). Social workers within multidisciplinary teams can reframe negative reactions and behaviors as normal reactions for this developmental stage (Jones \& Weisenfluh, 2003). Social worker's expertise in bio-psycho-social-spiritual assessments and treatment planning provides the interdisciplinary team with a comprehensive understanding of the AYA's needs and strengths and how to integrate these into a holistic treatment plan (NASW, 2004). Social work values and abilities in leadership through encouraging collaboration, respecting diversity, and resolving conflict make them particularly valuable on interdisciplinary teams (Remke \& Schermer, 2012).

The social work profession can play a critical role in services to AYA and their families grounded in a holistic and person-centered framework. Social workers can also transfer this framework to advocating for patients and their families on interdisciplinary teams, facilitating open communication, and 
ensuring all the patient's bio-psycho-social-spiritual needs are assessed and addressed in a developmentally appropriate and strengths-based manner. Despite the many advantages the social work profession has in serving this population, there is significantly less literature published in social work journals when compared to other professions working in this setting. It is important that the social work profession is an active contributor to the scientific research community in order to further the development of knowledge and best prac-tices, through the lens of a well-rounded and strengths-based perspective.

\section{REFERENCES}

Association for Children with Life-Threatening or Terminal Conditions and their Families. (2003). A guide to the development of children's palliative care services. Bristol, UK: publisher.

Bakke, A. (2016). Empowering our youth: Initiating sexual health education on the inpatient unit for the chronically ill pediatric patient. Urologic Nursing, 36(6), 267-275.

Barr, R. D., Ferrari, A., Ries, L., Whelan, J., \& Bleyer, W. A. (2016). Cancer in adolescents and young adults: A narrative review of the current status and a view of the future. JAMA Pediatrics, 170(5), 495-501. doi:10.1001/jamapediatrics.2015.4689

Bates, A. T., \& Kearney, J. A. (2015). Understanding death with limited experience in life: Dying children's and adolescents' understanding of their own terminal illness and death. Current Opinion in Supportive and Palliative Care, 9(1), 40-45. doi: 10.1097/ $\underline{\text { SPC. } 0000000000000118}$

Beaune, L., Leavens, A., Muskat, B., Ford-Jones, L., Rapoport, A., Shaul, R. Z., ... Chapman, L. A. (2014). Poverty and pediatric palliative care: What can we do? Journal of Social Work in EndOf-Life \& Palliative Care, 10(2), 170-185. doi:10.1080/15524256.2014.906375

Belasco, J., Danz, P., Drill, A., Schmid, W., \& Burkey, E. (2000). Supportive care: Palliative care in children, adolescents, and young adults - model of care, interventions, and cost of care: A retrospective review. Journal of Palliative Care, 16(4), 39-46.

Bell, C., Skiles, J., Pradhan, K., \& Champion, V. (2010). End-of-life experiences in adolescents dying with cancer. Supportive Care in Cancer, 18(7), 827-835. doi:10.1007/s00520-009-0716-1

Bell, C. J., Skiles, J., Pradhan, K., \& Champion, V. L. (2010). End-of-life experiences in adolescents dying with cancer. Supportive care in cancer, 18(7), 827-835.

Bosma, H., Johnston, M., Cadell, S., Wainwright, W., Abernethy, N., Feron, A., ... Nelson, F. (2010). Creating social work competencies for practice in hospice palliative care. Palliative Medicine, 24(1), 79-87. doi:10.1177/0269216309346596

Brown, M. R., \& Sourkes, B. (2006). Psychotherapy in pediatric palliative care. Child and Adolescent Psychiatric Clinics of North America, 15(3), 585-596. doi:10.1016/j. chc.2006.02.004

Carter, B., Qualter, P., \& Dix, J. (2015). Social relationships, loneliness and adolescence: The potential for disruption by chronic illness. Journal of Child Health Care, 19(4), 421-422. doi: $\underline{10.1177 / 1367493515618477}$ 
Clark, J. K., \& Fasciano, K. (2015). Young adult palliative care: Challenges and opportunities. American Journal of Hospice \& Palliative Medicine, 32(1), 101-111. doi:10.1177/ 1049909113510394

Cohen-Gogo, S., Marioni, G., Laurent, S., Gaspar, N., Semeraro, M., Gabolde, M., ... Brugières, L. (2011). End-of-life care in adolescents and young adults with cancer: Experience of the adolescent unit of the Institut Gustave Roussy. European Journal of Cancer, 47(18), 2735-2741. doi:10.1016/j.ejca.2011.09.008

Creswell, C., Christie, D., \& Boylan, J. (2001). Ill or adolescent? Developing group work on an adolescent medicine unit. Clinical Child Psychology and Psychiatry, 6(3), 351-362. doi: $10.1177 / 1359104501006003005$

Davies, B., Brenner, P., Orloff, S., Sumner, L., \& Worden, W. (2002). Addressing spirituality in pediatric hospice and palliative care. Journal of Palliative Care, 18(1), 59-67.

Davies, J., Kelly, D., \& Hannigan, B. (2015). Autonomy and dependence: A discussion paper on decision-making in teenagers and young adults undergoing cancer treatment. Journal of Advanced Nursing, 71(9), 2031-2040. doi:10.1111/jan.2015.71.issue-9

Derish, M. T., \& Heuvel, K. V. (2000). Mature minors should have the right to refuse lifesustaining medical treatment. The Journal of Law, Medicine \& Ethics: A Journal of the American Society of Law, Medicine \& Ethics, 28(2), 109-124. doi:10.1111/j.1748720X.2000.tb00001.x

Fahlberg, B. (2016). Integrating supportive and palliative care for young adults with serious illnesses. Nursing, 46(6), 12-14. doi:10.1097/01.NURSE.0000482877.08113.ab

Feudtner, C., Hays, R. M., Haynes, G., Geyer, J. R., Neff, J. M., \& Koepsell, T. D. (2001). Deaths attributed to pediatric complex chronic conditions: National trends and implica-tions for supportive care services. Pediatrics, 107(6), e99-e99. doi:10.1542/peds.107.6.e99 Foster,

T. L., Bell, C. J., \& Gilmer, M. J. (2012). Symptom management of spiritual suffering in pediatric palliative care. Journal of Hospice \& Palliative Nursing, 14(2), 109-115. doi:10.1097/NJH.0b013e3182491f4b

Fraser, J., Harris, N., Berringer, A., Prescott, H., \& Finlay, F. (2010). Advanced care planning in children with life-limiting conditions - the Wishes Document. Archives of Disease in Childhood, 95(2), 79-82. doi:10.1136/adc.2009.160051

Freyer, D. R. (2004). Care of the dying adolescent: Special considerations. Pediatrics, 113(2), 381-388. doi:10.1542/peds.113.2.381

Geiger, A. M., \& Castellino, S. M. (2011). Delineating the age ranges used to define adolescents and young adults. Journal of Clinical Oncology, 29(16), e492-e493. doi:10.1200/JCO.2011.35.5602

Greydanus, D. E. (2016). Caring for the dying adolescent. International Journal of Child and Adolescent Health, 9(3), 281.

Grinyer, A., \& Thomas, C. (2004). The importance of place of death in young adults with terminal cancer. Mortality, 9(2), 114-131. doi:10.1080/13576270310001659436

Hinds, P. S., Burghen, E. A., \& Pritchard, M. (2007). Conducting end-of-life studies in pediatric oncology. Western Journal of Nursing Research, 29(4), 448-465. doi:10.1177/ $\underline{0193945906295533}$

Hølge-Hazelton, B., Timm, H. U., Graugaard, C., Boisen, K. A., \& Sperling, C. D. (2016). "Perhaps I will die young." Fears and worries regarding disease and death among Danish adolescents and young adults with cancer. A mixed method study. Supportive Care in Cancer, 24(11), 4727-4737. doi:10.1007/s00520-016-3322-Z

Hughes, R. E., Holland, L. R., Zanino, D., Link, E., Michael, N., \& Thompson, K. E. (2015). Prevalence and intensity of pain and other physical and psychological symptoms in adolescents and young adults diagnosed with cancer on referral to a palliative care 
service. Journal of Adolescent and Young Adult Oncology, 4(2), 70-75. doi:10.1089/ jayao.2014.0015

Jacobs, S., Perez, J., Cheng, Y. I., Sill, A., Wang, J., \& Lyon, M. E. (2015). Adolescent end of life preferences and congruence with their parents' preferences: Results of a survey of adolescents with cancer. Pediatric Blood \& Cancer, 62(4), 710-714. doi:10.1002/pbc.25358

Janisch, M., Nolte-Buchholtz, S., \& von der Hagen, M. (2016). What kind of general and special palliative care do children, adolescents, and young adults with Duchenne muscular dystrophy need? Neuropediatrics, 47(S 01), P04-P08. doi:10.1055/s-0036-1583653

Jones, B. (2005). Pediatric palliative and end-of-life care: The role of social work in pediatric oncology. Journal of Social Work in End-Of-Life \& Palliative Care, 1(4), 35-62. doi: $10.1300 / \mathrm{J} 457 \mathrm{v} 01 \mathrm{n} 0404$

Jones, B., \& Weisenfluh, S. (2003). Pediatric palliative and end-of-life care: Developmental and spiritual issues of dying children. Smith College Studies in Social Work, 73(3), 423443. doi: $10.1080 / 00377310309517695$

Jones, B. L., Christ, G., \& Blacker, S. (2006). Companionship, control, and compassion: A social work perspective on the needs of children with cancer and their families at the endof-life. Journal of Palliative Medicine, 9(3), 774-788. doi:10.1089/jpm.2006.9.774

Kazmerski, T. M., Weiner, D. J., Matisko, J., Schachner, D., Lerch, W., May, C., \& Maurer, S. H. (2016). Advance care planning in adolescents with cystic fibrosis: A quality improvement project. Pediatric Pulmonology, 51(12), 1304-1310. doi:10.1002/ppul.23559

Kelly, J. A., May, C. S., \& Maurer, S. H. (2016). Assessment of the spiritual needs of primary caregivers of children with life-limiting illnesses is valuable yet inconsistently performed in the hospital. Journal of Palliative Medicine, 19(7), 763-766. doi:10.1089/jpm.2015.0509

Kirk, S., \& Fraser, C. (2014). Hospice support and the transition to adult services and adulthood for young people with life-limiting conditions and their families: A qualitative study. Palliative Medicine, 28(4), 342-352. doi:10.1177/0269216313507626

Klepping, L. (2012). Total pain: A reflective case study addressing the experience of a terminally ill adolescent. International Journal of Palliative Nursing, 18(3), 121-127. doi:10.12968/ijpn.2012.18.3.121

Knapp, C., Quinn, G. P., Murphy, D., Brown, R., \& Madden, V. (2010). Adolescents with lifethreatening illnesses. The American Journal of Hospice \& Palliative Care, 27(2), 139-144. doi: $\underline{10.1177 / 1049909109358310}$

Knox, M. K., Hales, S., Nissim, R., Jung, J., Lo, C., Zimmermann, C., \& Rodin, G. (2017). Lost and stranded: The experience of younger adults with advanced cancer. Supportive Care in Cancer, 25(2), 399-407. doi:10.1007/s00520-016-3415-8

Linebarger, J. S., Ajayi, T. A., \& Jones, B. L. (2014). Adolescents and young adults with lifethreatening illness: Special considerations, transitions in care, and the role of pediatric palliative care. Pediatric Clinics of North America, 61(4), 785-796. doi:10.1016/j. pcl.2014.05.001

Lyon, M. E., Jacobs, S., Briggs, L., Cheng, Y. I., \& Wang, J. (2014). A longitudinal, randomized, controlled trial of advance care planning for teens with cancer: Anxiety, depression, quality of life, advance directives, spirituality. Journal of Adolescent Health, 54(6), 710717. doi:10.1016/j.jadohealth.2013.10.206

Mack, J. W., Cannavale, K., Sattayapiwat, O., Cheung, B., Chen, L. H., Cooper, R. M., \& Chao, C. R. (2016). Care in the final month of life among adolescent and young adult cancer patients in Kaiser Permanente Southern California. Journal of Palliative Medicine, 19(11), 1136-1141. doi:10.1089/jpm.2015.0527

Marris, S., Morgan, S., \& Stark, D. (2011). 'Listening to Patients': What is the value of ageappropriate care to teenagers and young adults with cancer? European Journal of Cancer Care, 20(2), 145-151. doi:10.1111/ecc.2011.20.issue-2 
McAliley, L., Hudson-Barr, D., Gunning, R., \& Rowbottom, L. (2000). The use of advance directives with adolescents. Pediatric Nursing, 26(5), 471-482.

McNeil, S. B. (2016). Spirituality in adolescents and young adults with cancer: A review of literature. Journal of Pediatric Oncology Nursing, 33(1), 55-63. doi:10.1177/ $\underline{1043454214564397}$

Morgan, S., Davies, S., Palmer, S., \& Plaster, M. (2010). Sex, drugs, and rock 'n'roll: Caring for adolescents and young adults with cancer. Journal of Clinical Oncology, 28(32), 48254830. doi:10.1200/JCO.2009.22.5474

Muirhead, P. (2004). When parents and physicians disagree: What is the ethical pathway? Paediatrics \& Child Health, 9(2), 85-86. doi: $10.1200 / J C O .2009 .22 .5474$

National Association of Social Workers. (2004). NASW standards for social work practice in palliative and end-of-life care. Washington, DC: Author.

Osgood, D. W., Foster, E. M., \& Courtney, M. E. (2010). Vulnerable populations and the transition to adulthood. Future of Children, 20(1), 209-229. doi:10.1353/foc.0.0047

Pritchard, S., Cuvelier, G., Harlos, M., \& Barr, R. (2011). Palliative care in adolescents and young adults with cancer. Cancer, 117(S10), 2323-2328. doi:10.1002/cncr.26044

Quinn, G. P., Gonçalves, V., Sehovic, I., Bowman, M. L., \& Reed, D. R. (2015). Quality of life in adolescent and young adult cancer patients: A systematic review of the literature. Patient Related Outcome Measures, 6, 19-51. doi:10.2147/PROM.S51658

Remke, S. S., \& Schermer, M. M. (2012). Team collaboration in pediatric palliative care. Journal of Social Work in End-Of-Life \& Palliative Care, 8(4), 286-296. doi:10.1080/ $\underline{15524256.2012 .735551}$

Stern, M., Krivoy, E., Foster, R. H., Bitsko, M., Toren, A., \& Ben-Arush, M. (2010). Psychosocial functioning and career decision-making in Israeli adolescent and young adult cancer survivors. Pediatric Blood \& Cancer, 55(4), 708-713. doi:10.1002/pbc.22642

Stillion, J. M., \& Papadatou, D. (2002). Suffer the children: An examination of psychosocial issues in children and adolescents with terminal illness. American Behavioral Scientist, 46 (2), 299-315. doi: $10.1177 / 000276402236679$

Wein, S., Pery, S., \& Zer, A. (2010). Role of palliative care in adolescent and young adult oncology. Journal of Clinical Oncology, 28(32), 4819-4824. doi:10.1200/JCO.2009.22.4543 Wiener, L. (2012). Allowing adolescents and young adults to plan their end-of-life care. Pediatrics for Parents, 28(7/8), 28-29.

Wiener, L., Ballard, E., Brennan, T., Battles, H., Martinez, P., \& Pao, M. (2008). How I wish to be remembered: The use of an advance care planning document in adolescent and young adult populations. Journal of Palliative Medicine, 11(10), 1309-1314. doi:10.1089/ jpm.2008.0126

Wiener, L., Weaver, M. S., Bell, C. J., \& Sansom-Daly, U. M. (2015). Threading the cloak: Palliative care education for care providers of adolescents and young adults with cancer. Clinical Oncology in Adolescents and Young Adults, 51(18), 1-30. doi:10.2147/COAYA. $\underline{\mathrm{S} 49176}$

Winters, D. E., \& Beerbower, E. (2017). Mindfulness and meditation as an adjunctive treatment for adolescents involved in the juvenile justice system: Is repairing the brain and nervous system possible? Social Work in Health Care, 56(7), 615-635. doi: 10.1080/ $\underline{00981389.2017 .1316341}$

Zadeh, S., Pao, M., \& Wiener, L. (2015). Opening end-of-life discussions: How to introduce Voicing My CHOiCES ${ }^{\mathrm{TM}}$, an advance care planning guide for adolescents and young adults. Palliative \& Supportive Care, 13(3), 591-599. doi:10.1017/S1478951514000054 